\title{
Accurate Delivery of Online Advertising and the Evaluation of Advertising Effect Based on Big Data Technology
}

\author{
Tao Ge ${ }^{1}$ and Xiaolong $\mathrm{Wu}$ iD $^{2}$ \\ ${ }^{1}$ College of Humanities and Communication, Shandong Technology and Business University, Yantai 264005, Shandong, China \\ ${ }^{2}$ College of Culture and Art, Chengdu University of Information Technology, Chengdu 610103, Sichuan, China \\ Correspondence should be addressed to Xiaolong Wu; wuxiaolong@cuit.edu.cn
}

Received 2 July 2021; Revised 4 August 2021; Accepted 7 August 2021; Published 17 August 2021

Academic Editor: Sang-Bing Tsai

Copyright (c) 2021 Tao Ge and Xiaolong Wu. This is an open access article distributed under the Creative Commons Attribution License, which permits unrestricted use, distribution, and reproduction in any medium, provided the original work is properly cited.

\begin{abstract}
With the rapid development of the global online advertising industry, computational advertising was born. As an advertising model that combines technology and calculation with online advertising decision-making, it is based on a given advertising request, takes into account the advertising context and user characteristics, selects the best match from the potential advertising library through calculation, and then runs deliver ads to target audiences. This article aims to start from the technical level of big data, accurately place online advertisements, and evaluate the effects of advertisements. The sample collection method and statistical analysis method are used to collect samples and set up a data transmission system based on BD-MQ. The experimental results of the research show that there is almost no profit in the study of advertising time between 4 and 8 o'clock, and the users who watched at 16 o'clock reached $34.7 \%, 18-20$ o'clock reached $68.7 \%, 20-22$ o'clock reached $80.1 \%$ of the peak, and $22-24$ o'clock returned to $40.2 \%$. Compared with the data transmission system based on ActiveMQ, the data transmission system based on BD_MQ designed in the thesis has a speed advantage of about $5 \%-10 \%$, and the transmission rate changes for a single data with different sizes are more stable. The larger the total amount of data transmitted, the higher the average rate; the larger the single data packet, the higher the average rate. When the size of a single data packet is around $100 \mathrm{kB}$, the transmission rate reaches the maximum. The research on the precise placement and effect evaluation of online advertising has been completed well.
\end{abstract}

\section{Introduction}

The rapid development of Internet technology is having a huge impact on traditional industries, and it also brings new opportunities and challenges to traditional industries [1]. Online advertising is a good example of Internet technology challenging the traditional media industry. Traditional media refers to newspapers, magazines, radio, and television, but now the network marketing method with network advertising as the main medium is gradually gaining market share of traditional media, and network advertising has become a common form of advertising in people's daily life. According to the 2020 annual monitoring report of China's online advertising industry by iResearch, China's online advertising revenue in 2019 is approximately 646.43 billion yuan, exceeding 600 billion RMB. In 2019, China's mobile ads revenue was approximately 541.52 billion yuan. The scale of the short video industry is about 130.24 billion yuan. It can be seen that the mobile advertising market is still the mainstay of China's online advertising market. It can be seen that online advertising is gradually becoming the center of the advertising market. In the next few years, with the maturity and development of mobile Internet technology, the scale of online advertising still has strong growth potential.

The theoretical significance of the research object of this article lies in: exploring the operating mechanism of users using their own network influence to spread advertising effects and using literature reading, mathematical modeling, and experimental simulation to analyze the elements of the influence of network influence users and at the same time measuring the effect of online advertising. Based on the 
constructed input and output indicators, a preference dynamic DEA model is established. Based on the calculated value, suitable influential users for the enterprise are selected. Through the game model, prices for the selected advertising spokespersons are set. The results of this research can enrich the knowledge of network marketing management theory systems and provide theories and support methods for enterprises' social marketing management decision-making.

Wang and Lin developed a hybrid model that first uses a gradient boosting decision tree to transform the features of many input features, then uses the transformed features as the input of a logarithmic regression linear classifier, and finally obtains an impression of the result of the estimated hit ratio. They got a good result. However, his research did not use time window technology and could not track changes in users' interests over time [2]. Chae et al. of IBM Research Institute invented the relational database in the early 1970s. The emergence of this database became a milestone in the history of world software development, and it was also the starting point for the development of big data processing technology. But his research did not further plan the next development direction of big data processing technology [3]. In order to solve the above problems, Jiang constructed a DEA evaluation model of neutral cross efficiency, thereby eliminating the choice problem of the above two models. However, the final performance value calculated by the arithmetic mean value in his research is not Pareto equilibrium and still has great limitations [4].

The innovations of this article are as follows. (1) The addition of experimental data from both the algorithm and the actual advertising survey, combining theory and practice, makes the results of the article more convincing. (2) A logistic regression model based on dichotomy was added to the research on the precise placement of online advertisements. The introduction and simplification of the algorithm using the maximum likelihood estimation method greatly increase the intelligence of the research object of advertising. (3) Consumer psychology is researched, and the effect evaluation of advertising is studied from the perspective of consumers.

\section{Method of Accurate Placement of Online Advertising and Its Advertising Effect Evaluation Based on Big Data Technology}

2.1. Enterprise Positioning in the Advertising Process. Before formulating precision marketing strategies, companies should first have a relatively accurate positioning of the company and the products to be marketed [5]. No product of any company can satisfy all consumers, so the company is required to have a positioning for this product of the company, that is to say, it has its own sales group and sales scope, which is precisely the target of the product. Enterprises need to fully understand the advantages and gaps between their products and other similar products, lock the uniqueness of their products, and integrate them into the overall process of product development, production, and sales $[6,7]$.
Before advertising, the advertisement must also be positioned. The company accurately locates the target audience on the basis of an accurate analysis of the target population's psychological behavior [8] and then taps the target audience's most important factors for automotive products based on the target audience's psychological characteristics. And the company uses this as a basis to design advertisements, chooses appropriate ways to deliver customized advertisements that can meet the needs of the target audience [9], and ensures that the recipients can recognize and understand the content of the advertisement, so as to remember its brand and products [10] that can ultimately affect consumers' purchasing decisions. Accurate advertising positioning will undoubtedly have a positive and successful effect on the implementation and realization of the company's overall advertising planning strategy [11]. There are four steps for companies to achieve precise positioning of online advertising and marketing: first, companies should make accurate judgments on the characteristics and needs of the audience they face [12]; second, they must be able to position clearly and accurately the value they can provide; third, they must establish the value of precise communication; and fourth, they must accurately deliver value [13], as shown in Figure 1.

Consumers are a scattered but huge group, which exists in every corner of society [14]. Different regions, ages, consumption habits, cultural environments, and other factors have created different consumer groups [15]. Therefore, when targeting passenger car advertising groups, it is necessary to be able to target the target audience as accurately as possible [16] and place the passenger car product advertisements in the right place at the right time, so that potential consumers can see [17] and convert them into real consumers as much as possible $[18,19]$.

Relying on big data provides convenience for companies to accurately locate target audience [20]. Figure 2 shows the process of companies achieving precise target audience positioning.

2.2. Online Advertising Pricing. Online advertising pricing is one of the important issues of current online marketing [21]. Reasonable advertising pricing not only can promote the development of the advertising industry but also can reduce the advertising costs of enterprises and promote the dissemination of advertising information [22]. Advertising pricing has become a key element for the success or failure of the enterprise's network marketing strategy. At present, based on the evaluation criteria of online advertising effects, pricing, and revenue sharing theories [23], two types of advertising pricing models based on process and output have gradually formed, such as CPM (cost per mill) and CPT (cost per time) and CPC (cost per click) and CPS (cost per sale). CPM and CPT take the process as the pricing benchmark. As long as the advertisement is exposed, regardless of its effect [24], advertisers need to pay the advertisement publisher's fee [25]. CPC and CPS use the output as the pricing benchmark [26], and advertisers only pay for clicks or commodity transactions [27]. However, compared with traditional online advertising promotion methods, social 


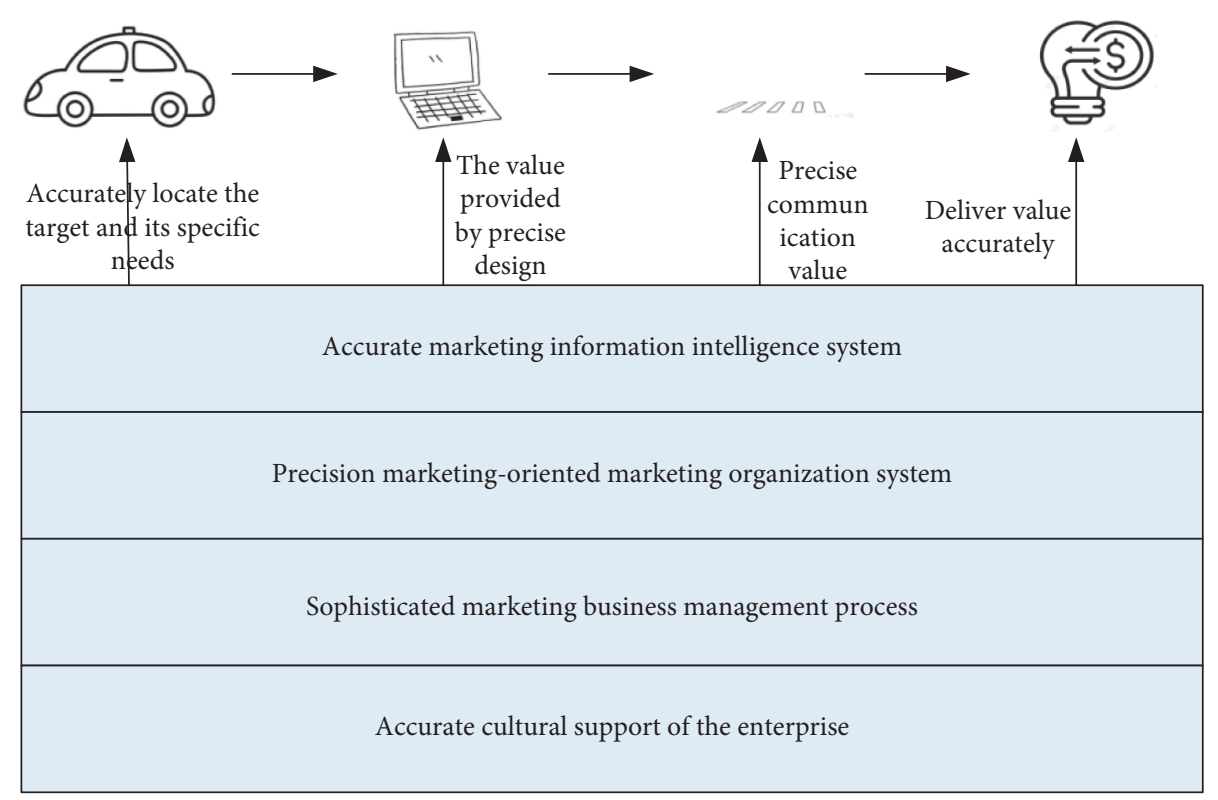

FIgURE 1: Steps for precise positioning of enterprises.

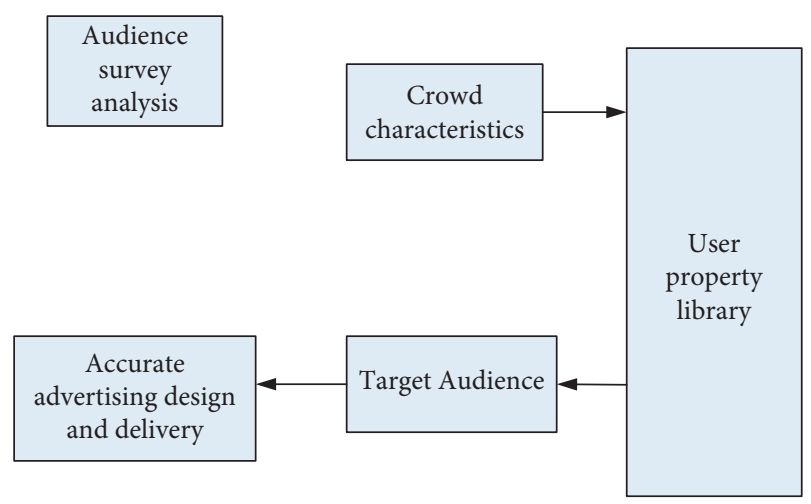

FIgURe 2: Audience targeting process.

networks provide users with an information interaction platform where users can browse, forward, comment, and like information [28]. Multiple interactive methods greatly enrich the advertising effect. At the same time, traditional advertising pricing methods are also difficult to meet the evaluation needs of enterprises. Therefore, it is necessary to take into account the characteristics of social networking platforms and formulate appropriate advertising pricing plans for them.

The current research on the use of influential users to place advertisements by enterprises is insufficient. In this new social network advertising model, how to choose to use influential users as corporate advertising spokespersons and conduct reasonable advertising pricing for this behavior has become the key to advertising research.

In the research on the use of influential network users, some scholars measure the influence of the network and choose the most influential network users as the advertising spokesperson. Other scholars trace the appropriate advertising spokespersons by measuring the effect of advertising. However, neither of these two methods comprehensively considers the comprehensive utility of advertising input and advertising output. The selected advertising spokesperson is not sufficient, and it is difficult to maximize the advertising effect. Therefore, it is necessary to find a comprehensive evaluation standard to consider advertising performance. At the same time, the advertising performance measurement model should be able to satisfy the user relationship complexity, data dynamics, and preferences of social networks. It has to be ensured that the calculated advertising performance value is reasonable and effective.

How to set appropriate advertising pricing for advertising spokespersons not only helps companies reduce advertising expenditures but also effectively motivates advertising spokespersons and further expands the value of advertising performance. The traditional advertising pricing model mainly starts from the advertising effect and makes appropriate advertising pricing based on CPM, CPC, and other methods. However, in social networks, this pricing method seems unreasonable. First of all, social networks can provide a variety of interactive data, which is difficult to meet the traditional click-through rate. Second, influential users can be used to place advertisements, and a certain amount of advertising deposit is to be paid in advance. Only through the final advertising effect pricing, it is difficult to form an incentive effect. Therefore, it is urgent to explore the different stages of advertising pricing under the new model. The best advertising pricing for the company has to be found through the study of different stages.

2.3. Logistic Regression Model. Logistic regression is a classification algorithm in machine learning. Due to the simplicity and efficiency of the algorithm, it has a wide range of applications in the industry. In the actual problems related to online advertising, all the problems we may encounter can be solved by the two-category algorithm.

The training dataset written in the question is as follows: 


$$
T=\left\{\left(x^{1}, y^{1}\right),\left(x^{2}, y^{2}\right), \ldots,\left(x^{i}, y^{i}\right)\right\},
$$

where $x^{i}$ is an $n$-dimensional vector, $y^{i}$ takes a value in $\{0,1\}, 1$ represents a positive example, and 0 represents a negative example. follows:

The hypothetical function of logistic regression is as

$$
h_{\theta}(x)=g\left(\theta^{T} x\right)=\frac{1}{1+e^{-\theta^{T} x}} .
$$

Logistic regression is based on the following assumptions:

$$
\begin{aligned}
& P(y=1 \mid x ; \theta)=h_{\theta}(x), \\
& P(y=0 \mid x ; \theta)=1-h_{\theta}(x),
\end{aligned}
$$

where $P(y=1 \mid x ; \theta)$ represents the probability that the output $y$ corresponding to the input $x$ under the parameters is 1 , and $P(y=0 \mid x ; \theta)$ is the probability that the output is 0 , which can also be written as follows:

$$
P(y \mid x ; \theta)=\left(h_{\theta}(x)\right)^{y}\left(1-h_{\theta}(x)\right)^{1-y} .
$$

The solution of logistic regression lies in how to obtain the parameters $\theta$. Here, maximum likelihood estimation is used. The idea of maximum likelihood estimation believes that the parameters of the model should be the set of parameters that maximize the likelihood of the training set data. In the logistic regression model, the likelihood is as follows:

$$
L(\theta)=\prod_{i=1}^{N} P\left(y^{i} \mid x^{i} ; \theta\right)=\prod_{i=1}^{N}\left(h_{\theta}\left(x^{i}\right)\right)^{y^{i}}\left(1-h_{\theta}\left(x^{i}\right)\right)^{1-y^{i}} .
$$

By taking the logarithm on both sides, we can get the following formula:

$$
L(\theta)=\log L(\theta)=\sum_{i=1}^{N} y^{i} \log h\left(x^{i}\right)+\left(1-y^{i}\right) \log \left(1-h\left(x^{i}\right)\right) .
$$

By using the stochastic gradient ascent method to solve, the following parameter update formula can be obtained:

$$
\theta_{j}:=\theta_{j}+\alpha\left(y^{i}-h_{\theta}\left(x^{i}\right)\right) x_{j}^{i},
$$

where $\alpha$ is the update step size, which can control the convergence speed of the parameter solution.

After obtaining the parameters, we can calculate the probability that the classifier will judge it as a positive example under the feature vector $x$ through the given feature vector $x$ as follows:

$$
P(y=1 \mid x ; \theta)=\frac{1}{1+e^{-\theta^{T} x}} .
$$

After selecting an appropriate threshold value, the classifier judges an instance with a probability value greater than this value as a positive example and an instance with a probability value less than this value as a negative example.
The training data set with a weight distribution of $D_{m}$ can be used to learn, and the basic classifier can be obtained as follows:

$$
G_{m}(x): X \longrightarrow\{-1,+1\} .
$$

The classification error rate calculated on the training set is as shown in the following formula:

$$
e_{m}=\sum_{i=1}^{N} w_{m, i} I\left(G_{m}\left(x_{i}\right) \neq y_{i}\right)
$$

In summary, the introduction of the algorithm is complete, and the experiment is ready to begin.

\section{Experiment on the Precise Placement of Online Advertising and Its Advertising Effect Evaluation Based on Big Data Technology}

3.1. Experimental Goals and Datasets. The goal of the experiment in this section is to analyze the effectiveness of the above features and evaluate the impact of abnormal user detection on the prediction of ad click-through rate. This section uses the ad click logs for a total of three weeks from July 4 to 24,2020 . The experimental data in this section is a preprocessed ad click dataset.

The true-positive rate (TPR) represents the percentage of positive samples that are correctly classified in the total positive samples, and the false-positive rate (FPR) represents the percentage of negative samples that are misclassified in the total negative samples. The curve drawn with FPR as the abscissa and TPR as the ordinate is the ROC (receiver operating characteristic) curve, and the area under the curve is called AUC (area under curve).

AUC is often used to evaluate the pros and cons of a binary classifier. When the binary classifier obtains the classification probability value on the test sample set, a threshold is selected. The binary classifier will judge the instances with the classification probability greater than this threshold as positive and the instances with the classification probability less than this threshold as negative. For example, comparing the classification results on the test sample set with the true values, the FPR and TPR values under the selected threshold can be obtained. In the same way, when different thresholds are selected, different (FPR and TPR) pairs will be generated, so a curve passing through $(0,0)$ and $(1,1)$ can be obtained, which is the ROC curve of the binary classifier.

In the experiment, the web crawler tool GooSeeKer was used in the "Self-driving Tour" section to capture the user data of the ad-posting users and the audience and store them in the .xlsm file. The data items captured are as follows: $X 1$ represents the user's personal identity, which is derived from the authentication of the number of cars owned by the user on the Autohome website (hereinafter referred to as the website). There are two authentication methods: plus " $V$ " authentication and ordinary authentication. " $V$ " certification is more difficult than ordinary certification, so the weight of " $V$ " certification is 2 and the weight of ordinary 
certification is 1 . That is, the value of personal identity $=$ the number of certified cars with " $V$ " $* 2+$ the number of ordinary certified cars $* 1 ; X 2$ represents the user's rank prestige, derived from the user's rank value on the website; $X 3$ represents the quality of posting, and the data come from the network user on the website. Publish the weighted sum of the number of text words and the number of pictures in the advertisement post. Considering that pictures have a higher interpretation effect than text, a weight of 50 is assigned to the pictures, that is, the post quality = the number of text words + the number of pictures $* 50 ; X 4$ represents the number of posts, and the data come from the sum of the number of posts posted by website users since the date of registration; $X 5$ represents the number of replies, and the data come from the sum of the number of posts posted by website users since the date of registration; X6 represents the frequency of interaction, and the data come from user participation. The sum of the number of interactive badges awarded after the site's interactive activities; $Y 1$ represents the number of clicks on the advertising posts, and the data come from the number of clicks on the advertising posts published by the website users within a week; Y2 represents the number of other people's replies to the advertising posts, the data come from the number of advertisement posts posted by website users and the number of replies from other users in a week; $Y 3$ represents the response quality of the advertisement post. The data are derived from the total number of words and the number of replies by other users within a week of the total number of words posted by a website user's advertisement post, that is, the reply quality of the advertisement post = the total number of words in the reply post/the number of reply posts.

3.2. Sample Selection and Observation Period. Because the DEA model has restrictions on the number of advertising decision-making units DMU, the number of DMUs must be greater than or equal to twice the sum of input and output indicators. Therefore, the data collection work of this experiment started at 12 o'clock on April 8, 2020; the posts published by all users in the observed section of the forum on that day was downloaded; the interference posts and zombie posts that are not related to the advertising theme were removed, and then the posts were filtered out. Twentythree users and their advertising posts that day were used as research samples.

The time span of the empirical study is six months; the data observation record is every Monday at 10 o'clock in the evening; and the extracted observation values are stored in the .xlsm file. By comparing the data of each month, this article found that the activity of advertising posts dropped rapidly in the fourth month. The advertising effect data of most influential network users tend to be 0 , so this article chooses the data of the previous four months as the research object of this article. Since DMU's social network advertising output effects have different units, it will increase the difficulty of comparison. Therefore, this article standardizes the three indicators of advertising output effects. Finally, the method of equal weight integration is used to calculate the coefficient of advertising effect in each period. Taking advertising coverage as an example, this article standardizes the advertising effects of each influential network user in the first stage and then calculates the arithmetic average of the advertising coverage in the first stage. From this, the average of the other two indicators of advertising output and the arithmetic average of the above three indicators are calculated. Finally, the social network advertising effect coefficient is obtained in the first stage. As can be seen from the Figures 1 and 2, the vertical axis of the article represents the effect of social network advertising, ranging from 0 to 1 ; the horizontal axis represents the effect of advertising. The larger the advertising effect coefficient, the more active the advertising posts at this stage.

3.3. The Social Effects of Online Advertising. In the era of big data, many new roles have been added to the original Internet advertising ecosystem. For example, in the RTB industry chain, in addition to advertisers, there are DSP, DMP, ad exchange platforms, and SSPs. These roles have not only changed the advertising operation model but also triggered a change in the traditional business-thinking model. The importance of data and technology has been noticed and valued, and the mode of thinking has changed in the new era, contributing to the social progress of society.

Advertising will have a certain impact on people's consumption behavior and social value orientation when conveying information. In the era of big data, online advertising has realized the personalization of advertising services, based on individual needs, and fully embodies the people-oriented concept. The advertising model has been transformed from a "disturbance type" to a "service type," meeting the needs of the audience in real time and efficiently, reflecting a humanistic caring spirit.

With big data, online advertising achieves precise targeting of target audiences and accurate placement of advertisements, which effectively solves the problem of waste of resources in traditional advertising models. It is a systematic process. Real-time accurate control of delivery status and effects requires a complete advertising delivery management platform, real-time monitoring, timely feedback, and real-time correction.

\section{Precise Placement of Online Advertising and Its Advertising Effect Evaluation Based on Big Data Technology}

4.1. Online Advertising Time and Evaluation System. After choosing the right content, the right way of advertising should also be placed in the right time, so that more audiences can click and browse the advertisements, and the high efficiency of clicks should be ensured. Different groups of people have different surfing habits. For example, young office workers prefer to surf the Internet at night, and students surf the Internet during holidays. With the popularization of the mobile Internet, people prefer to use the fragmented time such as waiting, riding, and resting to browse information online. According to the survey, users' 
online time in daily life is mainly concentrated between 18 and 24 o'clock, of which $20-22$ o'clock is the peak of the Internet throughout the day. Figure 3 shows the specific online time distribution.

It can be seen from Figure 3 that if the advertising time is placed at $4-8$ o'clock, the effect of advertising is basically ineffective. This requires that the company must be based on the specific advertising object, advertising budget, expected advertising effect, and competition. According to the situation of the advertisers, the specific schedule of advertising is scheduled to ensure that the target audience of the advertising can be received.

Due to the diversified forms of online advertising, different forms of advertising are also very different, and the effects produced are also different. For example, compared to text-only advertising forms, video ads can attract the attention of audiences. Therefore, when evaluating the effect of advertising, it is necessary to assign different points for evaluation according to the different forms of advertising.

The page browsing time that needs to be calculated is the time that the network user actually browses on the web page, not including the minimized or closed state of the web page.

Average browsing time refers to calculating the average of the time spent by all network users browsing a certain page. In the calculation process, the page browsing time is quantified, and different scoring standards are set for different lengths of time to display more intuitively the effect of online advertising.

The average browsing time indicator of the advertisement is to be selected as the revised indicator of awareness. The time the user stays on the advertisement is positively correlated with his awareness of the advertisement.

According to the analysis, the calculation and correction indexes of each evaluation dimension are determined, as shown in Table 1:

The impact of each information on the advertising effect has to be analyzed, and the weight configuration from the overall evaluation system has to be considered. In real life, the number of visits is used to evaluate attention, the number of clicks is used to evaluate awareness, the number of customer interactions is used to evaluate the degree of interaction, and the number of customer purchases is used to evaluate the degree of action.

For the weights in the calculation, the weights are different due to different industries, so it is necessary to use the consulting method to determine the index weights. Advanced technology should be used to monitor the effects of advertisements in real time, provide timely feedback to advertisers, make timely corrections, and further realize the precise placement of advertisements.

4.2. Function Test of Data Transmission System. During data communication, the node transmission module and the central synchronization module of the data transmission system perform data transmission and reception at the same time. When the transmission node receives the data sent by the sender, the central synchronization module always receives the data from the transmission node. As long as there

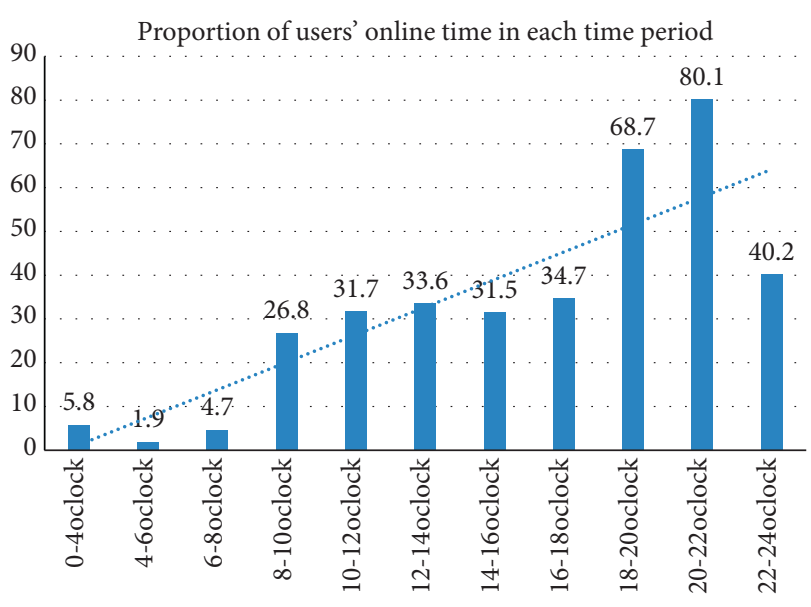

Weights

Figure 3: Proportion of users' online time in each time period.

TABLE 1: The index system of evaluating the effect of online advertising.

\begin{tabular}{lc}
\hline Evaluation dimension & Basic and reference indicators \\
\hline Attention A & Visits/thousands, PV \\
Ad slot, A1 \\
Ad format, A2 \\
Average page view time, A3 \\
\hline Awareness C & Ad clicks, AC \\
\hline Interaction I & Advertising average view time, C1 \\
\hline Action degree B & Number of customer interactions, CI \\
\hline
\end{tabular}

is new data in the node, the central synchronization module will automatically synchronize to the central synchronization module.

In the following test, in order to find out the value of the number of concurrent connections, the message transmission speed reaches the highest and tends to be stable. Table 2 and Figure 4 show the results of the node transmission data test.

However, due to the limitations of the table, the performance of the node data transmission test is not good. When the node transmits data to microseconds, the connection speed drops significantly, as shown in Figure 4.

Table 3 shows the results of the synchronization data rate test of the central server.

After adding the digital variable, the test result of the central server synchronization data rate is shown in Figure 5.

It can be seen from Table 2 and Figure 5 that the overall trend of the central synchronization system is roughly the same as that of the node receiving system, but when persistent connections are used, the speed is slower than the transmission speed of nodes. On the contrary, when nonpersistent connections are used, the speed is slower than that of nodes. The transmission speed should be faster.

According to the above test of data transmission and data collection of the transmission system, it can be found that the use of persistent connection technology has great advantages in throughput, but this advantage begins to 
TABLE 2: Statistics of the number of data packets received by the node per second.

\begin{tabular}{lcc}
\hline Number of concurrent connections & Persistent connection & Nonpersistent connection \\
\hline 1 & 6794 & 3602 \\
5 & 25987 & 12198 \\
50 & 92487 & 27345 \\
100 & 114936 & 27879 \\
500 & 117978 & 29164 \\
1000 & 119874 & 28687 \\
\hline
\end{tabular}

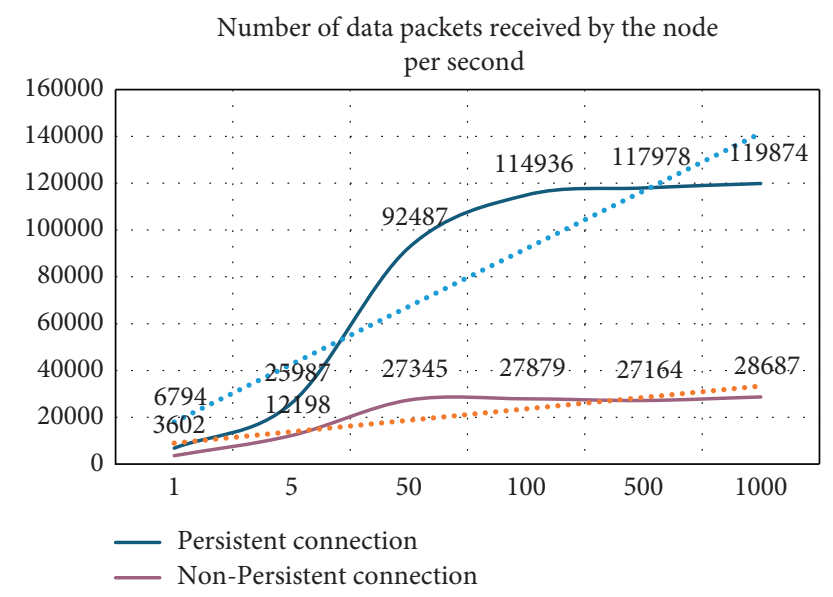

FIGURE 4: Number of data packets received by the node per millisecond.

TABLE 3: Statistics on the number of data packets received by the center per second.

\begin{tabular}{lcc}
\hline Number of concurrent connections & Persistent connection & Nonpersistent connection \\
\hline 1 & 8072 & 4214 \\
5 & 29268 & 15341 \\
50 & 61987 & 28648 \\
100 & 65781 & 28684 \\
500 & 95261 & 31321 \\
1000 & 94978 & 33415 \\
\hline
\end{tabular}

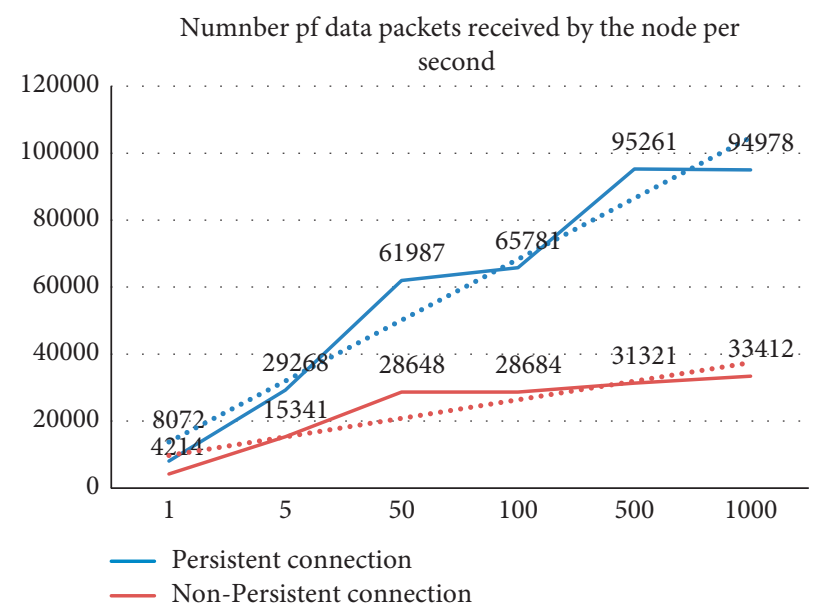

Figure 5: Number of data packets received by the node per second. 
TABLE 4: ActiveMQ dual thread sending rate.

\begin{tabular}{lcccc}
\hline \multirow{2}{*}{ Single data volume } & \multicolumn{5}{c}{ Total amount of data } \\
& $0.1 \mathrm{M}$ & $1 \mathrm{M}$ & $10 \mathrm{M}$ & $100 \mathrm{M}$ \\
\hline $0.1 \mathrm{kB}$ & 0.19 & 1.49 & 13.18 & - \\
$1 \mathrm{kB}$ & 0.07 & 0.45 & 2.01 & 14.32 \\
$10 \mathrm{kB}$ & 0.04 & 0.14 & 1.66 & 8.45 \\
$100 \mathrm{kB}$ & 0.01 & 0.06 & 0.47 & 5.48 \\
$1 \mathrm{MB}$ & - & 0.13 & 0.81 & 7.60 \\
$10 \mathrm{MB}$ & - & - & 0.49 & 4.37 \\
\hline
\end{tabular}

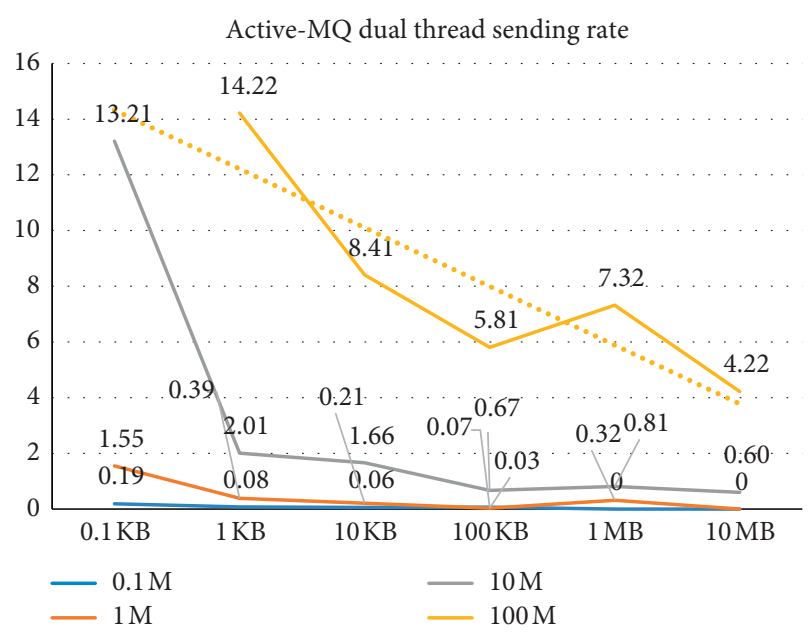

FIGURE 6: Data sending rate test result of ActiveMQ transmission system after adding dummy variables.

TABLE 5: BD_MQ dual thread transmission rate.

\begin{tabular}{lcccc}
\hline \multirow{2}{*}{ Single data volume } & \multicolumn{5}{c}{ Total amount of data } \\
& $0.1 \mathrm{M}$ & $1 \mathrm{M}$ & $10 \mathrm{M}$ & $100 \mathrm{M}$ \\
\hline $0.1 \mathrm{kB}$ & 0.21 & 1.36 & 12.09 & - \\
$1 \mathrm{kB}$ & 0.11 & 0.42 & 3.04 & 12.77 \\
$10 \mathrm{kB}$ & 0.07 & 0.16 & 1.96 & 8.01 \\
$100 \mathrm{kB}$ & 0.02 & 0.08 & 0.48 & 5.42 \\
$1 \mathrm{MB}$ & - & 0.19 & 0.91 & 7.47 \\
$10 \mathrm{MB}$ & - & - & 0.54 & 4.02 \\
\hline
\end{tabular}

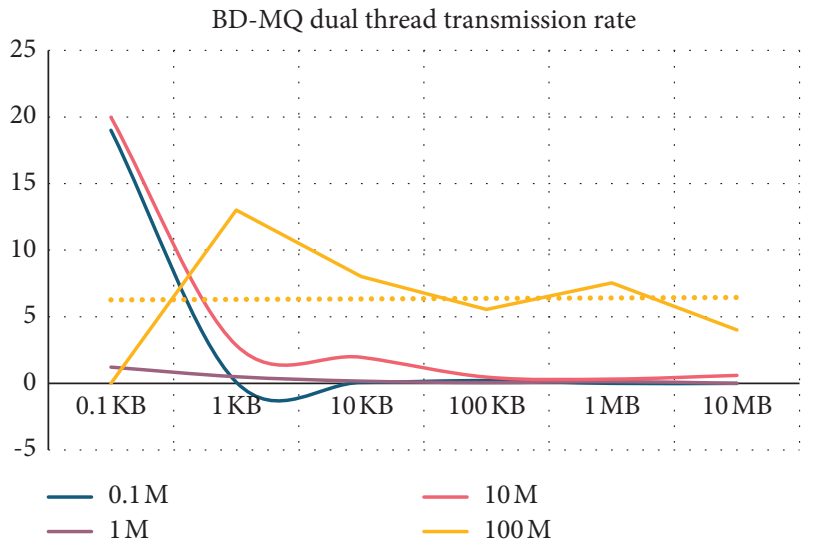

Figure 7: BD_MQ dual thread transmission rate. decrease as the number of concurrent connections exceeds 500. This is because long connections are often long. Time occupies the communication connection and hinders the access of other concurrent threads. When the number of concurrent connections is about 500, the throughput can reach the maximum.

4.3. Data Transmission Throughput Comparison Test. The entire transmission system is developed based on the above communication management module, BD_MQ and BD_MC, so when testing the data transmission performance, it needs to be compared with other MQ-based transmission systems. Here, ActiveMQ is selected for comparison testing.

The test uses dual threads, with two servers as the node side, and selects $0.1 \mathrm{kB}, 1 \mathrm{kB}, 10 \mathrm{kB}, 100 \mathrm{kB}, 1 \mathrm{MB}$, and $10 \mathrm{MB}$, a total of 6 data specifications as the single transmission data volume, and also selects $0.1 \mathrm{MB}, 1 \mathrm{MB}, 10 \mathrm{MB}$, and $100 \mathrm{MB}$, these 4 data totals to observe the difference in the transmission time between the two.

Table 4 shows the results of the data transmission rate test of the ActiveMQ transmission system.

After adding the dummy variable, the test result of the data-sending rate of the ActiveMQ transmission system is shown in Figure 6.

Table 5 is the test results of the data-sending rate of the BD_MQ transmission system.

The data-sending rate test result of the BD-MQ transmission system after adding dummy variables is shown in Figure 7.

According to the above test results, it can be seen that the data transmission system based on BD_MQ designed in the paper has a speed advantage of about 5\%-10\% compared with the data transmission system based on ActiveMQ, and the transmission rate changes for different sizes of single data more stable. The larger the total amount of data transmitted, the higher the average rate; the larger the single data packet, the higher the average rate. When the size of a single data packet is around $100 \mathrm{kB}$, the transmission rate reaches the maximum.

\section{Conclusions}

The experimental results show that compared with the current traditional advertising and effect evaluation, the accurate delivery of online advertisements based on big data technology and the evaluation of advertising effects proposed in this paper are faster; the audiences are more accurate; and the advertising investment is faster. This article uses a logistic regression model and data statistics method to set up a data transmission system based on BD_MQ. The experimental results show that: in the study of advertising time, there is almost no profit between 4 and 8 points, and the users who watched at 16:00 reached $34.7 \%, 18-20$ points reached $68.7 \%, 20-22$ points reached $80.1 \%$ of the peak value, and at 22-24 returned to $40.2 \%$. Compared with the data transmission system based on ActiveMQ, the data transmission system based on BD_MQ designed in the thesis 
has a speed advantage of about $5 \%-10 \%$, and the transmission rate changes for a single data with different sizes are more stable. The larger the total amount of data transmitted, the higher the average rate; the larger the single data packet, the higher the average rate. When the size of a single data packet is around $100 \mathrm{kB}$, the transmission rate reaches the maximum. The research on the precise placement and effect evaluation of online advertising has been completed well. The shortcomings of this experiment are as follows. (1) It did not consider the scientificity and rationality of the construction of indicators such as network influence and advertising effect. An introduction to this type of indicator can be added in future research. (2) The logistic regression model used in the calculation algorithm is only considered at the level of dichotomy, and no other methods are adopted. More algorithmic methods can be used to demonstrate in future research.

\section{Data Availability}

The data that support the findings of this study are available from the corresponding author upon reasonable request.

\section{Conflicts of Interest}

The authors declare that there are no potential conflicts of interest with respect to the research, authorship, and/or publication of this article.

\section{Acknowledgments}

This work was supported by the Key R\&D Program of Shandong Province (soft science project) General items 2020RKO1558.

\section{References}

[1] C.-H. Wu, Z. Yan, S.-B. Tsai, W. Wang, B. Cao, and X. Li, “An empirical study on sales performance effect and pricing strategy for E-commerce: from the perspective of mobile information," Mobile Information Systems, vol. 2020, Article ID 7561807, 8 pages, 2020 .

[2] H. Wang and C. Lin, "Research on effect evaluation of online advertisement based on resampling method," Mathematical Problems in Engineering, vol. 2020, Article ID 4617581, 15 pages, 2020.

[3] I. Chae, H. A. Bruno, and F. M. Feinberg, "Wearout or weariness? Measuring potential negative consequences of online ad volume and placement on website visits," Journal of Marketing Research, vol. 56, no. 1, pp. 57-75, 2019.

[4] Y. Jiang, "Research on the best visual search effect of logo elements in internet advertising layout," Journal of Contemporary Marketing Science, vol. 2, no. 1, pp. 23-33, 2019.

[5] X. Chen and F. Zhang, "Evaluation of news communication effect based on cognitive neuroscience," Translational Neuroscience, vol. 10, no. 1, pp. 31-36, 2019.

[6] H. Zhou, X. Gu, and L. Li, "The dynamic investment strategy of online advertising based on spillover effect in duopoly competition market," Computing, vol. 100, no. 8, pp. 881-905, 2018.

[7] C.-H. Wu and S.-B. Tsai, "Using DEMATEL-based ANP model to measure the successful factors of E-commerce,"
Journal of Global Information Management, vol. 26, no. 1, pp. 120-135, 2018.

[8] M.-Y. Chang, K.-M. Cho, and S.-H. Park, "Research of advertising effect about brand fitness class advertisement using SNS: focusing on regulatory focus and construal level," Journal of Sport and Leisure Studies, vol. 68, no. 4, pp. 103118, 2017.

[9] R. G. Duffett, "Effect of instant messaging advertising on the hierarchy-of-effects model amid teenagers in South Africa," The Electronic Journal of Information Systems in Developing Countries, vol. 72, no. 1, pp. 1-21, 2016.

[10] K. Chu, D.-H. Lee, and J. Y. Kim, "The effect of non-stereotypical gender role advertising on consumer evaluation," International Journal of Advertising, vol. 35, no. 1, pp. 106134, 2016.

[11] Z. Ke, "The effect of para-social interaction in endorsement advertising: SEM studies based on consumers' exposure to celebrity symbols," Language and Semiotic Studies, vol. 6, no. 1 , pp. 46-81, 2020.

[12] H. j. Li and M. Peng, "Online course learning outcome evaluation method based on big data analysis," International Journal of Continuing Engineering Education and Life-Long Learning, vol. 29, no. 4, pp. 349-361, 2019.

[13] M. Hamouda, "Understanding social media advertising effect on consumers' responses: an empirical investigation of tourism advertising on Facebook," Journal of Enterprise Information Management, vol. 31, no. 2, pp. 12-40, 2018.

[14] Z. Zhang, Y. Zhou, X. Xie et al., "Research on advertising click-through rate estimation based on feature learning," Chinese Journal of Computers, vol. 39, no. 4, pp. 780-794, 2016.

[15] A. Kc, B. Dhl, and Y. Ji, "The effect of verbal brand personification on consumer evaluation in advertising: internal and external personification-ScienceDirect," Journal of Business Research, vol. 99, no. 7, pp. 472-480, 2019.

[16] V. Kumar, R. Raman, and R. Meenakshi, "Online advertising strategies to effectively market a business school," International Journal of Higher Education, vol. 10, no. 4, pp. 61-67, 2021.

[17] Q. He and H. Qu, "The impact of advertising appeals on purchase intention in social media environment-analysis of intermediary effect based on brand attitude," Journal of Business Administration Research, vol. 7, no. 2, pp. 17-21, 2018.

[18] J. Parra-Arnau, J. P. Achara, and C. Castelluccia, "My ad choices: bringing transparency and control to online advertising," ACM Transactions on the Web, vol. 11, no. 1, pp. 94-97, 2016.

[19] Z. Lv, L. Qiao, and I. You, "6G-enabled network in box for internet of connected vehicles," IEEE Transactions on Intelligent Transportation Systems, 2020.

[20] D. Belanche, C. Flavián, and A. Pérez-Rueda, "Understanding interactive online advertising: congruence and product involvement in highly and lowly arousing, skippable video ads," Journal of Interactive Marketing, vol. 37, no. 3, pp. 75-88, 2017.

[21] J. Jankowski, P. Kazienko, J. Wątróbski, A. Lewandowska, P. Ziemba, and M. Zioło, "Fuzzy multi-objective modeling of effectiveness and user experience in online advertising," $E x$ pert Systems with Applications, vol. 65, no. 12, pp. 315-331, 2016.

[22] M. Granfield and I. Mcardle, "Content marketing versus product marketing: an analysis of consumer engagement in 
online advertising," Journal of Digital Banking, vol. 1, no. 2, pp. 169-176, 2016.

[23] J.-Y. Jung, Y.-J. Shin, S.-C. Sohn et al., "Quantitative evaluation of patient-specific quality assurance using online dosimetry system," Journal of the Korean Physical Society, vol. 72, no. 2, pp. 312-319, 2018.

[24] S. Sridhar, F. Germann, C. Kang, and R. Grewal, "Relating online, regional, and national advertising to firm value," Journal of Marketing, vol. 80, no. 4, pp. 39-55, 2016.

[25] P. Hummel and R. P. McAfee, "Loss functions for predicted click-through rates in auctions for online advertising," Journal of Applied Econometrics, vol. 32, no. 7, pp. 1314-1328, 2017.

[26] B. Mintzes, "The tip of the iceberg of misleading online advertising comment on "trouble spots in online direct-toconsumer prescription drug promotion: a content analysis of FDA warning letters"," International Journal of Health Policy and Management, vol. 5, no. 5, pp. 329-331, 2016.

[27] M. Hanna and M. Honeychurch, "Chronic misleading online advertising by chiropractors," New Zealand Medical Journal, vol. 129, no. 1432, pp. 91-93, 2016.

[28] C. Moraes and N. Michaelidou, "Introduction to the special thematic symposium on the ethics of controversial online advertising," Journal of Business Ethics, vol. 141, no. 2, pp. 1-3, 2017. 